\title{
Current-Driven Monte Carlo Simulation of Conduction in Switching Chalcogenides
}

\author{
E. PiCCinini* And F. Buscemi \\ Advanced Research Center on Electronic Systems (ARCES) University of Bologna \\ Via Toffano 2/2, I-40125 Bologna, Italy
}

The $I(V)$ characteristics of amorphous chalcogenides usually show a negative differential conductance region, which makes the material switch from a high-resistive to a high-conductive state. This feature is of the utmost importance for adopting these materials in the manufacturing of solid-state memory devices. We propose here two complementary models for the interpretation of the switching mechanism, both stemming from and updating the literature analysis. The former is based on macroscopic equations that can be solved analytically; the latter is a current-driven three-dimensional Monte Carlo simulation of the device. A critical analysis of the two models is also performed in order to identify the fundamental conditions accounting for the voltage snap-back of the $I(V)$ curve.

PACS: 72.20.Ee, 72.20.Ht, 72.80.Ng

\section{Introduction}

The need for new, compact, high-density and reliable storage devices represents a challenge for modern electronics. Materials like chalcogenides, such as $\mathrm{Ge}-\mathrm{Sb}-\mathrm{Te}$ (GST) alloys, represent nowadays the standard technology for optical storage, due to the easiness to produce transitions between the $\mathrm{ON}$ and $\mathrm{OFF}$ states by means of a laser-controlled phase change. An analogous principle is applicable also within the solid-state memory technology, and non-volatile memories fully based on phase-change materials have recently been designed and are currently close to the market [1].

GST chalcogenides feature switching phenomena of two different kinds. The first one is thermally controlled and lets the material switch from the crystalline to the amorphous phase and vice versa. The second one, which is electrically controlled, involves only the amorphous phase, and is of the utmost importance in the device design. Even though this transition has widely been investigated in the past and some models have been proposed and discussed [2-5], a simulative framework able to reproduce this behavior and, eventually, suitable for incorporation into commercial device simulators, has not been released yet.

In this contribution we have tackled the problem with a twofold approach on a common background. At first, we have described the transport process by means of a one-dimensional model that can be solved analytically. The outcome of this first analysis has then led to the devel-

\footnotetext{
* corresponding author; e-mail: enrico.piccinini@unimore.it
}

opment of a three-dimensional Monte Carlo simulation, which is able to overcome some simplifying hypotheses that were initially assumed.

\section{The model}

Many investigations in the past demonstrated that the amorphous phase of GST is characterized by a number of microstructural defects acting as donor or acceptor traps [6-8]. This evidence suggests that an appropriate model for conduction in amorphous GST (a-GST) must include a trap-controlled process, like carrier hopping via localized states.

In order to investigate the switching phenomenon, the standard voltage-driven simulation must be turned into a current-driven one. It can be demonstrated [5] that when the electric potential across the GST region is monotonic and sufficiently steep, the trap-controlled current becomes independent of the potential and a saturation phenomenon occurs.

Here, the basic transport picture is enriched by adding a second contribution linked to electrons occupying extended states (or high-energy traps), and acting in parallel. By means of this addition the cited limit of the model described in [5] can be overcome.

\subsection{The macroscopic homogeneous model}

Concepts like conductance, mobility and carrier density can successfully be exploited to develop a one-dimensional model that describes the transport process as an average motion of carriers.

Let $n_{\mathrm{T}}$ and $n$ be the concentration of carriers in the traps and in the extended states, respectively. The latter carriers are free to move across the device, and may 
be scattered by the lattice. After a scattering event, depending on the energy loss, they may become trapped electrons, or remain in the extended states. Trapped electrons usually move from one trap to another one by thermally-assisted tunneling.

In general, the conduction $G_{\mathrm{C}}$ of the a-GST region can be expressed as the sum of two terms, the former referring to electrons in traps, the latter to electrons belonging to extended states

$$
G_{\mathrm{C}}=q(A / L)\left(\mu_{\mathrm{T}} n_{\mathrm{T}}+\mu n\right) .
$$

In (1), $\mu_{\mathrm{T}}$ and $\mu$ are the mobility of the trap and band carriers, $q$ is the electron charge, and $A$ and $L$ represent the cross-section and the length of the a-GST region, respectively. Even though $\mu \gg \mu_{\mathrm{T}}$, near the equilibrium condition $n$ is negligible, and the conductivity is dominated by the hopping process.

As the current increases, the voltage drop across the a-GST increases as well, and the upper edge of the potential profile is bent. Moreover, as the field grows, the tunneling distance shortens and the energy barrier separating two traps lowers, letting trap carriers hop more easily. Mobility $\mu_{\mathrm{T}}$ is thus a function of the electric field, so that the $I(V)$ characteristic can turn from an ohmic to a super-ohmic exponential behavior.

When the electric field is sufficiently high, field-emission phenomena also take place. Under these conditions, the probability that a trapped electron may tunnel out of the trap and reach a band state rapidly increases. In this way, the concentration $n$ grows, and the last summand in (1) gains more and more importance. Remembering that $\mu \gg \mu_{\mathrm{T}}$, this term gives origin to a high conductivity condition, and, eventually, allows for a decrease in the voltage drop across the a-GST (Fig. 1).

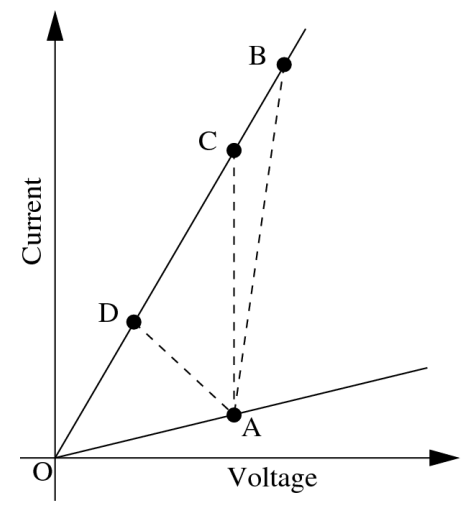

Fig. 1. Schematic $I(V)$ characteristic. If the conduction were due to trapped carriers or to extended states only, the characteristic would follow the $O A$ or the $O B$ lines, respectively. Three possible behaviors define the transition from the high-resistive to the high-conductive mode: a small increase of the potential associated to a wide transition interval ( $A B$ line), a hold condition with no increase of the potential ( $A C$ line), or a potential snap-back when the transition interval is sharp $(A D$ line).
Apparently, when the voltage drop lowers, field emission is weakened and, eventually, could be switched off. In order to make the whole process consistent one must assume a sustaining process as discussed in Sect. 3 .

\subsection{The Monte Carlo device simulation}

For numerical simulations, we have considered a simple device made of a small region of a-GST, sandwiched between two planar contacts. Inside the a-GST two sets $N_{\mathrm{T} 1}$ and $N_{\mathrm{T} 2}$ of randomly placed, donor-like traps exist. Traps are identical to each other, except for the intrinsic energy level, which is close to the midgap for $N_{\mathrm{T} 1}$ and close to the conduction band edge, about $0.3 \mathrm{eV}$ above of the midgap, for $N_{\mathrm{T} 2}$. The second set plays the role of the extended states of the macroscopic model. Contacts are modeled through virtual traps that can inject or host electrons at any time. The inclusion of a number of fixed negative compensating charges, not involved in the transport process, ensures the electrical neutrality.

The transition of a carrier from the trap $i$ to the trap $j$ is described by a combination of three processes. First, a phonon is absorbed and a virtual state $E^{*}$ is reached within trap $i$. The probability of such an event is proportional to the Boltzmann factor $\exp \left(\left(E_{i}-E^{*}\right) / k_{\mathrm{B}} T\right), k_{\mathrm{B}}$ and $T$ being the Boltzmann constant and temperature, respectively. Next, the electron leaves trap $i$ to reach trap $j$, tunneling through the energy barrier with probability $T_{i j}\left(E^{*}\right)$. Lastly, phonons are released until the carrier is trapped again in the energy level $E_{j}$ of trap $j$. This last event is assumed to always happen. We assume that if $E^{*}<E_{j}$ the transition does not occur. Summarizing, the transition probability $S_{i j}$ can be expressed by

$$
S_{i j}=\nu_{0} \int_{0}^{\infty} T_{i j}\left(E^{*}\right) \exp \left(\left(E_{i}-E^{*}\right) / k_{\mathrm{B}} T\right) \mathrm{d} E^{*},
$$

where $\nu_{0}$ is the attempt-to-escape frequency. $T_{i j}\left(E^{*}\right)$ is the transmission coefficient at energy $E^{*}$ for a double Coulomb well modeled by the electric field in between. The integral in (2) takes into account all possible virtual states, and is evaluated by means of Monte Carlo sampling.

Once the probabilities of each possible transition are known, two random numbers are generated, one to select the actual transition, and one to evaluate the time necessary for the hopping process. A full-detailed description of the Monte Carlo current-driven framework is reported in [5]. Here, we only remember the key-point: one must check whether the actual hop ends before an electron reaches the injection contact from the external circuit. If not, the transition is rejected and the procedure is cycled with the new configuration.

The shape of the energy profile separating the two sites $i$ and $j$, and, consequently, the transmission probability $T_{i j}$, strongly depend on the local field, as well as the trap energy levels. This asks for solving the Poisson equation self-consistently with the position of the carriers at each simulation step. It is a rather demanding task in terms of computational resources, and, in order to speed up the development of the model in its early stages, a 
simplified electrostatics was taken into account as described in [5]. The transmission probability $S_{i j}$ was thus evaluated according to the Miller and Abrahams expression [9]:

$$
S_{i j}=\left\{\begin{array}{c}
\nu_{0} \exp \left(r / r_{0}\right) \exp \left(\left(E_{i}-E_{j}\right) / k_{\mathrm{B}} T\right) \\
\text { if } E_{j}>E_{i} \\
\nu_{0} \exp \left(r / r_{0}\right) \\
\text { otherwise }
\end{array}\right.
$$

where $r_{0}$ is a characteristic localization length, defined by the overlap integral of the wave functions in the barrier region. For sufficiently low electric fields, a constant $r_{0}$ as in [9] is an acceptable approximation, but when high fields are applied the localization length must be calculated according to its definition, e.g. by means of the WKB approximation.

At present, the electrostatics is calculated using the finite-element method, which provides the potential profile inside the whole a-GST region, and, in turn, makes it possible to obtain $T_{i j}$ by calculating the total transfer matrix along the $i-j$ line.

\section{Results and discussion}

We report in Figs. 2 and 3 the $I(V)$ characteristics determined with the two models. More details specifying the parameters used in calculations are given in the captions.

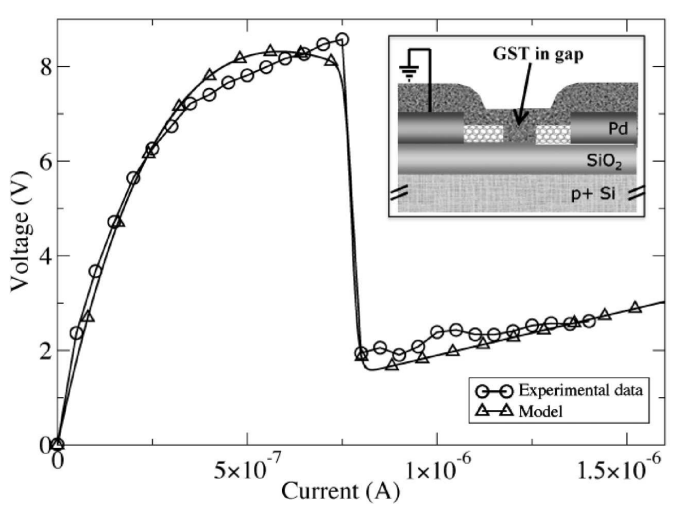

Fig. 2. Voltage vs. current characteristic determined with the macroscopic model. The parameters of the model have been fitted against experimental data obtained with a test device made of a $110 \mathrm{~nm}$ wide a-GST dome between two carbon nanotubes acting as electrodes, as sketched in the inset. More details can be found in [10]. Please not that abscissas refer to the current instead of the voltage.

The outcome of the Monte Carlo simulation, namely the trap occupation fraction, the average length and direction of the hops, and the potential profile across the a-GST region, allow for a microscopic interpretation of the switching phenomenon. As discussed in the following, this interpretation validates the assumptions of the macroscopic model.

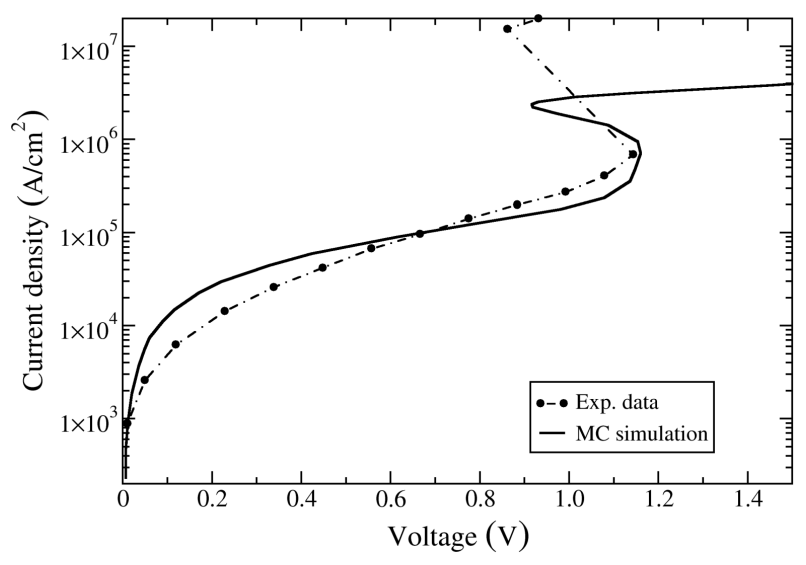

Fig. 3. Current density vs. voltage characteristic determined with the Monte Carlo simulation. A total trap density $N_{\mathrm{T}} \approx 1.5 \times 10^{19} \mathrm{~cm}^{-3}$ with energy around midgap has been used in a $28 \mathrm{~nm}$ long, $270 \mathrm{~nm}^{2}$ wide a-GST region; the voltage has been calculated as an average over 192 independent simulations to reduce the noise. The reference experimental data are taken from [4].

Depending on the value of the imposed current, three cases exist. When the current is low, the electric field is low and almost constant. This allows for a diffusive regime, where carriers can hop either in favor or against the direction imposed by the current with similar probability. The result of such a condition is the ohmic subthreshold conduction.

Increasing the current, a greater number of carriers accumulate on the injecting contact and leave the collecting one. As a consequence, a greater voltage drop across the a-GST is found. Since the transition rate depends on the field through both the height of the barrier and tunneling distance, a stronger field makes the transitions in the direction of the field itself more probable, along with longer hops. This imbalance between carriers going back and forth gives origin to the exponential subthreshold regime. We point out that in the subthreshold zone the high-energy traps stay empty for almost all of the time, as suggested by the macroscopic model.

When a critical field is reached, also the high-energy shallow traps become accessible in the region close to the collecting contact. Due to field effects, trapped carriers in the first half of the a-GST region can easily transfer to the second half, leaving a positive charge behind them. Conversely, a negative charge accumulates close to the collecting contact. The presence of these charge distributions influences the contribution generated by the traps to the potential profile, which has now a sudden drop in the region close to the injection contact, and a rise at the other edge. This initial drop allows for a continuous feed of the shallow traps in the second half of the device, making the process self-sustained. Depending on the balance between the charges accumulated on the contacts, the trap concentration and the internal charge dis- 
tribution, the overall effect on the total drop across the a-GST can either be a change in slope, or a sudden rise, or a snap-back, which are the three situations described by the macroscopic model. High currents are then sustained thanks to the shallow traps, which account for quick transitions across the whole a-GST region, as done in the macroscopic model by the extended states.

\section{Acknowledgments}

This work has been carried out under the Intel Corporation $34524 / 2009$ contract, whose support is gratefully acknowledged.

The authors would also like to thank Prof. Massimo Rudan, Prof. Rossella Brunetti and Prof. Carlo Jacoboni for the fundamental discussions and their precious suggestions.

\section{References}

[1] A. Pirovano, K. Shuegraph, Nature Nanotech. 5, 177 (2010).
[2] D. Adler, M.S. Shur, M. Silver, S.R. Ovshinsky, J. Appl. Phys. 51, 3289 (1980).

[3] A. Pirovano, A.L. Lacaita, A. Benvenuti, F. Pellizzer, R. Bez, IEEE Trans. Electron. Dev. 51, 452 (2004).

[4] D. Ielmini, Y. Zhang, J. Appl. Phys. 102, 054517 (2007); D. Ielmini, Phys. Rev. B 78, 03528 (2008).

[5] F. Buscemi, E. Piccinini, R. Brunetti, M. Rudan, C. Jacoboni, J. Appl. Phys. 106, 103706 (2009).

[6] C.B. Thomas, J. Phys. D 9, 2587 (1976).

[7] B.S. Lee, J.R. Abelson, S.G. Bishop, D. Kang, B. Cheong, K. Kim, J. Appl. Phys. 97, 093509 (2005).

[8] S. Caravati, M. Bernasconi, T.D. Kühne, M. Krack, M. Parrinello, J. Phys. Condens. Matter 21, 255501 (2009).

[9] A. Miller, E. Abrahams, Phys. Rev. 120, 745 (1960).

[10] M. Rudan, F. Giovanardi, T. Tsafack, F. Xiong, E. Piccinini, F. Buscemi, A. Liao, E. Pop, R. Brunetti, C. Jacoboni, in: Proc. SISPAD, Bologna 2010, IEEE 2010, p. 257. 